\title{
Is Periodontal Disease a Risk Factor for Recurrent Coronary Events?
}

\author{
Carmen loana Biriș ${ }^{1}$ Ioana Rodean²,3, Bogdan-Vasile Halațiu2,3, Theodora Benedek²,3 \\ 1 Faculty of Dental Medicine, "George Emil Palade" University of Medicine, Pharmacy, Science and Technology, Târgu Mureș, Romania \\ 2 Clinic of Cardiology, “George Emil Palade” University of Medicine, Pharmacy, Science and Technology, Târgu Mureș, Romania \\ ${ }^{3}$ Center of Advanced Research in Multimodality Cardiac Imaging, Cardio Med Medical Center, Târgu Mureș, Romania
}

\section{CORRESPONDENCE \\ loana Rodean \\ Str. Gheorghe Marinescu nr. 38 \\ 540139 Târgu Mureș, Romania \\ Tel: +40 265215551 \\ E-mail: ioana_patricia91@yahoo.com}

\section{ARTICLE HISTORY}

Received: September 13, 2021

Accepted: October 12, 2021
Carmen Biriș • Str. Gheorghe Marinescu nr. 38, 540139 Târgu Mureș, Romania. Tel: +40 265215 551, E-mail: biris.carmen@umfst.ro

Bogdan-Vasile Halațiu • Str. Gheorghe Marinescu nr. 38, 540139 Târgu Mureș, Romania. Tel: +40 265215 551, E-mail: bhalatiu@yahoo.com

Theodora Benedek • Str. Gheorghe Marinescu nr. 38 540139 Târgu Mureș, Romania. Tel: +40 265215 551, E-mail: theodora.benedek@gmail.com
In the current paper, we present the case of an adult male, aged 43 years, with recurrent acute coronary syndromes, who was admitted several times to the Cardiology Department of the Emergency County Clinical Hospital of Târgu Mureș, Romania. One year prior to the present admission, the patient underwent a coronary angiography due to myocardial infarction, which revealed an acute thrombotic stenosis on the right coronary artery. This lesion was treated according to the current guidelines with thrombectomy followed by the successful implantation of a drug-eluting stent.

At the current admission, the patient presented with typical angina at 6 hours after the onset. Physical examination revealed normal vital signs, blood pressure $100 / 60 \mathrm{mmHg}$ and pulse 70 beats per minute. Laboratory tests showed elevated levels of serum cardiac enzymes (classic I troponin $81.7 \mathrm{ng} / \mathrm{mL}$, creatin kinase 5,200 IU/L, and creatin kinase-MB 1,053 IU/L), normal lipid profile and high inflammatory markers (C-reactive protein $20 \mathrm{mg} / \mathrm{L}$, MMP-9 $287.63 \mathrm{ng} /$ $\mathrm{mL}$ ). The electrocardiography (ECG) revealed sinus rhythm with a frequency of 85 beats per minute, normal heart axis with an ST-segment elevation of 2-4 $\mathrm{mm}$ in leads V1-V6. Based on the patient's symptoms and paraclinical profile, the case was interpreted as anterior acute myocardial infarction with ST-segment elevation Killip class I, and an emergency coronary angiography was performed. The examination was well tolerated, without any complications, and showed restenosis in the previously implanted stent and the development of new, significant (greater than $70 \%$ ) coronary lesions in all three branches of the coronary tree. The culprit lesion was identified as an acute thrombotic occlusion of the anterior descending artery in the proximal segment, which was treated interventionally by thrombectomy followed by the implantation of a drug-eluting stent, with optimal result.

In order to ascertain the real origin of the accelerated atherosclerotic process in a young patient without significant comorbidities but with an important inflammatory profile, a complex dental examination was performed to assess the link between recurrent acute coronary syndrome and periodontal disease 


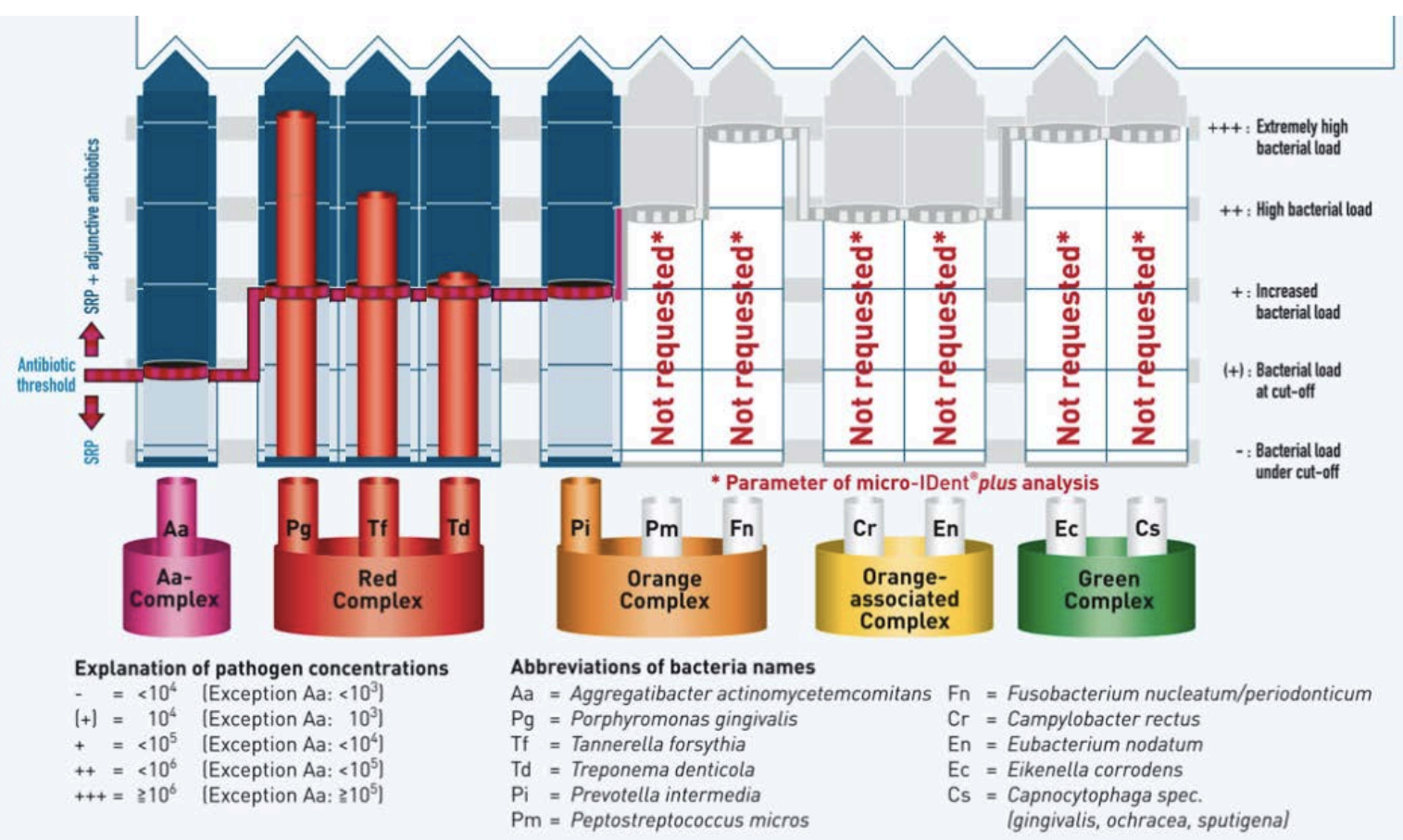

FIGURE 1. DNA test result for periodontopathogenic marker bacteria based on a next-generation sequencing technique

(PD). Given the presence of advanced PD, we decided to collect bacterial DNA samples from the gingival sites. The microbiological analysis showed a bacterial concentration requiring treatment due to the presence of germs from the red complex, especially an extremely high load of Porphyromonas gingivalis, the most frequent bacterium associated with aggressive PD (Figure 1).

Thus, during hospitalization, the patient received complex anti-ischemic therapy associated with an adjunctive antibiotic treatment with metronidazole $3 \times 400 \mathrm{mg} /$ day, for 7 days. At the one-month follow-up, the patient was stable, free of symptoms, with improved quality of life.

The patient agreed to the publication of his data, and the institution where the patient was admitted approved the publication of the case.

Acute coronary syndromes in young adults are often associated with risk factors such as smoking, dyslipidemia, diabetes mellitus etc. ${ }^{1}$ However, new literature data confirm also that inflammation plays a major role in the atherosclerotic process. One of the most frequent inflammatory disease is PD, remaining an important risk factor for recurrent acute coronary disease due to the possible association between this condition and plaque vulnerability. ${ }^{2,3}$ The presence of the PD is correlated with more severe cardiovascular disease and recurrences, with a poor outcome based on recent data. ${ }^{4}$ Thus, an accurate PD screening in patients with cardiovascular diseases would be of great im- portance for an early diagnosis and adequate treatment to reduce cardiovascular recurrences in young adults.

\section{CONFLICT OF INTEREST}

Nothing to declare.

\section{ACKNOWLEDGEMENT}

This review is part of the project entitled "Research on the interrelationship between periodontal disease and the degree of coronary atheromatous plaques-a study based on a modern panel of biological, inflammatory and imaging biomarkers" financed by the "George Emil Palade" University of Medicine, Pharmacy, Science and Technology, Târgu Mureș, contract number 615/3/17.01.2019.

\section{REFERENCES}

1. Iragavarapu T, Radhakrishna T, Babu KJ, Sanghamitra R. Acute coronary syndrome in young - A tertiary care centre experience with reference to coronary angiogram. J Pract Cardiovasc Sci. 2019;5:18-25.

2. Rodean IP, Lazăr L, Halațiu, VB, Biriș C, Benedek I, Benedek T. Periodontal Disease Is Associated with Increased Vulnerability of Coronary Atheromatous Plaques in Patients Undergoing Coronary Computed Tomography Angiography—Results from the Atherodent Study. J Clin Med. 2021;10:1290.

3. Fatima Z, Shahzadi C, Nosheen A, Khan M, Rehman HU. Periodontitis is a risk factor for developing cardiovascular diseases. J Pak Med Assoc. 2020;70:1941-1943.

4. Moras LL, Carvalho TA, Oliveira MB, et al. Impact of periodontal disease on late morbimortality (10 years) of patients with acute coronary syndrome. Int J Cardiovasc Sci. 2019;32:35-40. 